\title{
Embolización de arteria uterina en una paciente con sangrado vaginal y shock hipovolémico
}

\author{
Embolization of uterine artery in patient with vaginal bleeding \\ and hipovolemic shock
}

Diego Martín Barbetta', Miguel Osvaldo Villegas', Joaquín Ezequiel Etcheverre', Pablo Rojas², Pablo Nicolás Luna'.

\section{RESUMEN}

El sangrado vaginal masivo producido por un tumor cérvico uterino es una urgencia ginecológica, el tratamiento quirúrgico en el contexto de shock hipovolémico presenta una alta mortalidad. La angiografía permite localizar rápidamente el sitio de sangrado. Presentamos un caso de hemorragia vaginal severa resuelto con la embolización de la arteria uterina, con resultado exitoso y estabilización hemodinámica.

Palabras claves: hemorragia ginecológica, cáncer cervicouterino, shock hipovolémico, tratamiento endovascular percutáneo, embolización de arterias uterinas.

\section{ABSTRACT}

The massive vaginal bleeding produced by a cervical uterine tumor is a gynecological emergency, the surgical treatment in a hypovolemic shock has a high mortality. Angiography allows rapid localization of the bleeding site. We present a case of severe vaginal bleeding resolved with embolization of the uterine artery, with successful outcome and hemodynamic stabilization.

Keywords: gynecological hemorrhage, cervical cancer, hypovolemic shock, percutaneous endovascular treatment, uterine artery embolization.

Revista Argentina de Cardioangiología Intervencionista 2019;10(3):123-124. https://doi.org/10.30567/RACI/201903/0123-0124

\section{INTRODUCCIÓN}

La mayor parte de los casos descriptos de embolización de arterias uterinas de causas ginecológicas se deben a la hemorragia posparto, sin embargo, las primeras publicaciones de embolizaciones se debieron a hemorragias relacionadas a tumores malignos ${ }^{1,2}$. Está demostrado el beneficio del tratamiento endovascular en tumores cervicouterinos sangrantes en estadio avanzado ${ }^{3,45,6}$.

El enfoque quirúrgico de estos casos es complejo, debido al estadio avanzado de la enfermedad, las pacientes presentan un mal estado general y/o alteraciones pélvicas secundarias a la radioterapia o invasión del tejido neoplásico. La embolización resultaría beneficiosa como tratamiento paliativo para detener el sangrado y mejorar la calidad de vida de las pacientes.

Presentamos una paciente de 28 años con hemorragia vaginal masiva secundaria a tumor cervicouterino no estadificado, que no cedió con tratamiento médico, en shock hipovolémico, en la cual se decidió tratamiento endovascular, por la gravedad clínica y el consiguiente alto riesgo quirúrgico.

\section{CASO CLÍNICO}

Paciente de 28 años con tumor de cérvix en estudio, no estadificado al momento de la internación. Presentó episodios de hemorragia vaginal autolimitados. Ingreso por sangrado severo, se realizó taponamiento bajo especuloscopia en dos oportunidades con expulsión espontánea del tapón y persistencia de sangrado. Evolucionó con shock hipovolémico, se realizó tratamiento con vasopresores, transfusión de 7 unidades de glóbulos rojos (UGR), 6 unidades de plasma (UPF), expansión hídrica, ácido tranexámico y ácido aminocaproico.

Debido al alto riesgo quirúrgico, se decidió realizar angiografía con eventual embolización del vaso culpable.

1. Servicio de Hemodinamia y Cardiología Intervencionista

2. Servicio de Cirugía General. Hospital Municipal "San José," Exaltación de la Cruz, Buenos Aires

$\triangle$ Correspondencia:diegobarbe@gmail.com

Los autores no declaran conflictos de intereses

Recibido: 31/05/2019| Aceptado: 04/09/2019
El laboratorio previo al procedimiento fue Hto.: $19 \%, \mathrm{Hb}$ : 6,4 g/L, QUICK $15.5 \mathrm{seg}$, KPTT $25 \mathrm{seg}$, RIN 1,24, recuento de plaquetas 178.000 por $\mu \mathrm{l}$.

$\mathrm{Al}$ ingreso a la sala de Hemodinamia presentaba T.A.: 60/30 $\mathrm{mmHg}$, F.C: $136 \mathrm{lat} / \mathrm{min}$, con requerimiento de inotrópicos. Se colocó introductor $6 \mathrm{Fr}$ en arteria femoral derecha, crossover con catéter S1 e intercambio sobre cuerda 0,035 " por introductor mallado $60 \mathrm{~cm} .7 \mathrm{Fr}$ (Ansel Flexor Curva Multipropósito Cook Medical, Bloomington, U.S.A.) que se posicionó en arteria hipogástrica izquierda. Se realizó inyección control observando la arteria uterina izquierda en su recorrido vertical y sus ramas arcuatas que penetran el músculo uterino. Presenta imagen compatible con sangrado activo hacia cavidad (Figura 1). Se progresa catéter diagnóstico JR3 sobre cuerda hidrofílica 0.035" (Terumo Medical Corporation, Tokyo, Japón) selectivizando la arteria uterina. Se realizó embolización selectiva con esponja de gelatina (Spongostan, Johnson y Johnson Medical S.A., Nueva Jersey; U.S.A.) hasta la oclusión total de la rama. Se realiza control angiográfico bilateral, con resultado exitoso y sin signos de sangrado activo, dando por finalizado el procedimiento (Figura 2).

Durante la internación en área crítica se realizó transfusiones de UGR y UPF, intercurriendo con coagulopatía por consumo y trombocitopenia severa. Sin nuevo episodio de sangrado vaginal. Se logra corregir alteraciones hematológicas con 16 UGR, 14 UPF, 6 U de crioprecipitados de factor VIII y 6 U de plaquetas, con estabilización clínica y buena evolución. Permaneció 7 días en área crítica y 7 días en sala general.

Durante la internación se realizó biopsia de cérvix que informó carcinoma invasor moderadamente diferenciado con infección viral (HPV) concomitante.

En el control a los 30 días la paciente se encontraba estable y sin signos de sangrado activo vaginal.

La paciente se derivó a centro especializado en oncología para tratamiento de su enfermedad primaria.

\section{DISCUSIÓN}

Los tumores ginecoobstétricos son la segunda causa de cáncer femenino a nivel mundial. El sangrado severo vaginal es una de las 


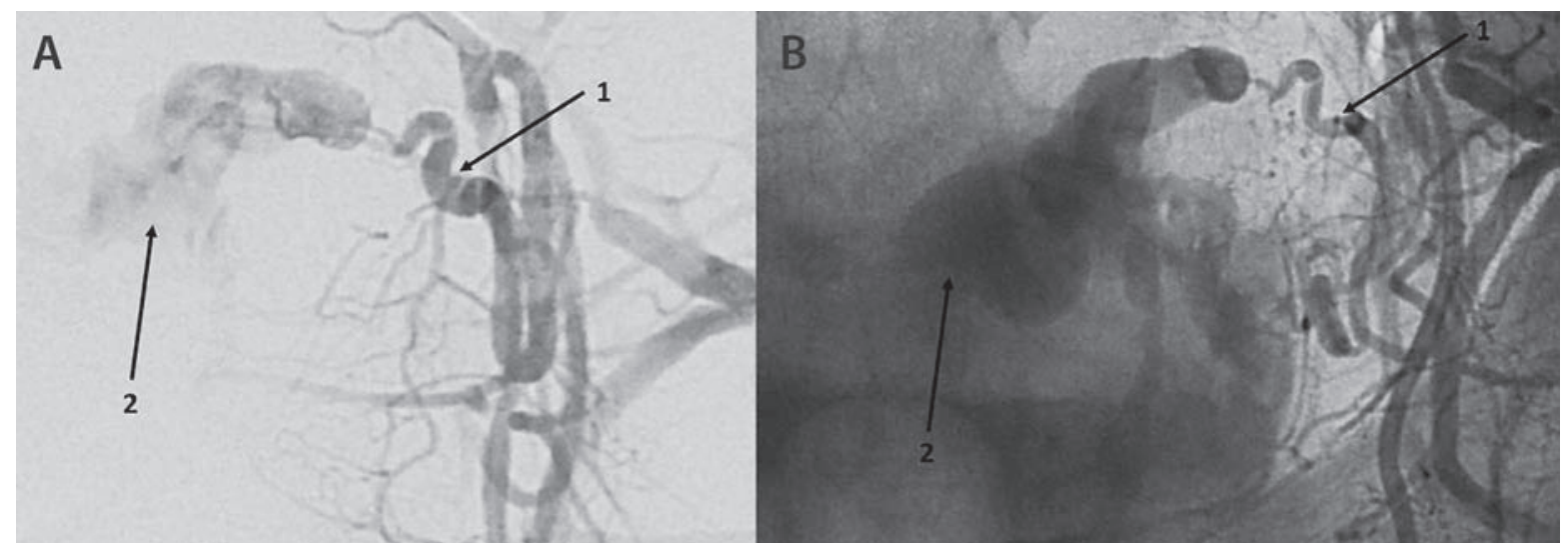

Figura 1. Angiografía selectiva de arteria Hipogástrica izquierda con (A) y sin sustracción (B). Se observa arteria uterina (1) con sangrado activo a cavidad uterina (2).

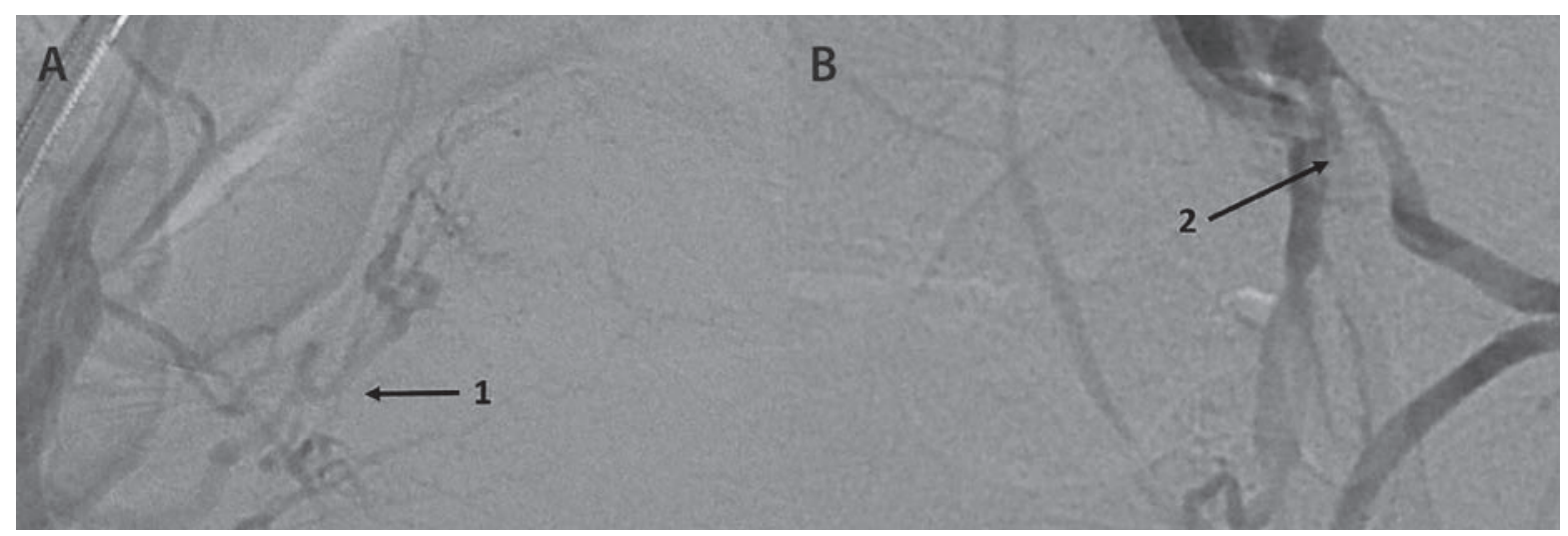

Figura 2. A. Arteria uterina derecha (1) de características normales. B. Arteria uterina izquierda ocluida posembolización (2).

complicaciones más graves que llevan a la paciente a un cuadro de anemia y/o shock hipovolémico con riesgo de vida. El tratamiento endovascular con embolización del vaso culpable es una opción rápida y efectiva, utilizando materiales embolizantes como esponja de gelatina, coágulo autólogo, coils, P.V.A. y microesferas. Los primeros casos descriptos fueron en la década del ' 70 con resultado exitoso en pacientes con tumores ginecológicos ${ }^{1,2}$.

En 108 pacientes con hemorragia severa por neoplasias pélvicas (50 en vejiga, 39 en útero, 16 en ovario y 3 en próstata), Pisco et al. embolizaron las arterias ilíacas internas logrando el éxito en el 89\% de los pacientes. El 64\% presentó náuseas, vómitos, dolor glúteo y fiebre debido a necrosis tisular ${ }^{3}$. Nuestra paciente no Presentó complicaciones posembolización relacionadas al procedimiento.

Yamashita et al. presentaron un éxito inmediato del 100\% en la embolización selectiva de la arteria uterina en 17 pacientes con sangrado severo y tumores malignos, a 3 pacientes los debió reintervenir ${ }^{4}$.

Mihmanlivet et al. presentaron el resultado exitoso en 6 pa- cientes (cuatro con carcinoma de cérvix, un carcinoma de endometrio y una metástasis vaginal de carcinoma de ovario) a quienes se realizó embolización con partículas de PVA5

Yalvac $S$ et al. describieron 6 casos de embolización selectiva de arterias uterinas en hemorragia incoercible en pacientes con cáncer cervicouterino en fase terminal, con éxito en todas ellas ${ }^{6}$. Nuestra paciente es un caso de hemorragia ginecológica severa secundario a tumor cervical, en shock hipovolémico, tratado por vía endovascular con embolización selectiva con esponja de gelatina, disponible en ese momento en nuestro servicio, de la arteria uterina izquierda, éxito inmediato y buena evolución a los 30 días.

\section{CONCLUSIÓN}

La embolización selectiva arterial en esta paciente con esponja de gelatina fue un procedimiento rápido, con bajo índice de comorbilidad, y puede ser una alternativa en el tratamiento del sangrado vaginal severo secundario a neoplasias cervicouterinas en pacientes con shock hipovolémico.

\section{BIBLIOGRAFÍA}

1. Miller FJ, Jr, Mortel R, Mann WJ, et al. Selective arterial embolization for control of hemorrhage in pelvic malignancy: femoral and brachial catheter approaches. AJR Am J Roentgenol. 1976;126:10281032.

2. Schwartz PE, Goldstein HM, WallaceS, et al. Control of arterial hemorrhage using percutaneous arterial catheter techniques in patients with gynecologic malignancies. Gynecol Oncol. 1975;3:276-288.

3. Pisco J M, Martins J M, Correia M G. Internal iliac artery: embolization to control hemorrhage from pelvic neoplasm. Radiology. 1989:172:337-339
4. Yamashita Y, Harada M, Yamamoto H, et al. Transcatheter arterialembolization of obstetric and gynaecological bleeding: efficacy and clinical outcome. Br J Radiol. 1994;67:530-534.

5. Mihmanlil,CantasdemirM, KantarciF,etal.Percutaneousembolizationinthemanagement of intractable vaginal bleeding. Arch Gynecol Obstet. 2001;264:211-4.

6. Yalvac S, Kayikcioglu F, Boran N, Tulunay G, et al. Embolization of uterine artery in terminal stage cervical cancers. Invest 2002;20(5-6):754-758

7. Jessica Nogueira-García, Rocío Moreno-Selva, María EstherRuiz-Sánchez, y col. Embolización de las arterias uterinas como tratamiento paliativo del cáncer de cérvix. Ginecol Obstet Mex 2015;83:289-293. 